\title{
An alternative medicine perspective on the role of the endocannabinoid system in the treatment of epilepsy
}

\begin{abstract}
The aim of this review is to evaluate the effects, both beneficial and adverse, of medical cannabinoids; and the role of the endocannabinoid system in epilepsy treatment in adults and children. Despite the introduction of new antiepileptic drugs, the therapeutic response for patients with epilepsy remains poor; and the quality of life using such drugs has not significantly improved. Notwithstanding several advantages, new pharmacological antiepileptic drugs have not satisfactorily reduced the number of refractory patients.

Cannabis has been used as a medicine for millennia. Current research has shown encouraging results for its use in the treatment of epilepsy. In cannabis, $\Delta 9$ tetrahydrocannabinol (THC) is the main psychoactive ingredient while cannabidiol (CBD) is the main non-psychoactive ingredient. THC has shown anticonvulsant properties in most animal models and many case reports. THC exerts its neural effects through the activation of $\mathrm{CB} 1$ receptors (CB1Rs) which are a part of the endocannabinoid system. Due to the psychotropic effects of THC, which have detrimental effects on cognition and behavior, its application for children and adolescents appear to be limited. To overcome this, the non-psychoactive compound, $\mathrm{CBD}$, is being researched. $\mathrm{CBD}$ has displayed anticonvulsant properties in numerous case reports, and in preclinical and clinical studies.

However, as yet, CBD does not have a completely understood mechanism of action. Numerous case reports, preclinical studies and clinical studies indicate that CBD and CBD-containing medications are able to help manage epilepsy in adults and children affected by refractory seizures, such as occur in Dravet syndrome and Lennox-Gastaut syndrome. Clinical trials are limited however. Although the data is promising, further preclinical and clinical studies are needed to better evaluate the potential therapeutic profile of CBD in epilepsy.
\end{abstract}

Keywords: cannabidiol, childhood epilepsy, endocannabinoid system, refractory form of epilepsy, $\delta 9$-tetrahydrocannabinol
Volume II Issue I - 2018

\author{
Inga Cicenaite Merz, ${ }^{1,3}$ Nicholas A Kerna, ${ }^{2}$ \\ Tony L Brown ${ }^{4}$ \\ Clear Lake Regional Hospital, USA \\ ${ }^{2}$ College of Medicine, University of Science, USA \\ Major General Hugh G. Robinson Center for Neuropsychiatric \\ Studies, USA \\ ${ }^{4}$ Harvard University, USA
}

Correspondence: Nicholas A Kerna, College of Medicine, University of Science, Art and Technology, 7583 Sourdough Dr, Morrison, CO 80465, USA, Email nicholas.kerna@usat.edu

Received: January 31, 2018 | Published: February I2, 2018
Abbreviations: CBD, cannabidiol; CB1R, CB1 cannabinoid receptor; $\mathrm{CB} 2, \mathrm{CB} 2$ cannabinoid receptor; $\mathrm{CW}$, charlotte's web; GPDR, G-protein coupled receptor; THC, $\Delta$ 9-tetrahydrocannabinol; 2-AG, 2-arachidonoyglycerol

\section{Introduction}

According to the American Epilepsy Society, more than three million Americans live with epilepsy. ${ }^{1}$ Availability of numerous antiepileptic drugs has not substantially reduced the proportion of patients with medically refractory epilepsy. Their quality of life and therapeutic response to currently available medications remains poor. Patients need new treatments that control seizures and have fewer side effects. This gap in epilepsy treatment-especially in refractory forms of childhood epilepsy has led patients to seek alternative treatments. To this purpose, emphasis has been given to phytocannabinoid treatment which has become more accepted. Phytocannabinoids are naturally occurring cannabinoids derived from the cannabis plant.

Researchers in many parts of the world are trying to understand how phytocannabinoids work, and how they might help in epilepsy.

\section{Discussion}

Cannabis sativa is one of world's most ancient medical plants. It has been used for over 5,000 years in many cultures for different therapeutic purposes. Marijuana has deeply American roots, too: George Washington, the first president of the United States, grew hemp on his estate at Mount Vernon. For most of America's history, cannabis was legal and was commonly found in tinctures and extracts. ${ }^{2}$ Marijuana's recreational use and abuse became common in Western societies in the 1960's, approximately the same time scientists started to look for explanations regarding the therapeutic and behavioral effects of Cannabis sativa.

The major psychoactive ingredient of cannabis was first isolated in 1964, and then identified as $\Delta 9$-tetrahydrocannabinol (THC) in $1967 .{ }^{3}$ Another major chemical component, structurally related to THC but non-psychoactive, cannabidiol (CBD), was identified in $1963 .{ }^{4}$ These discoveries launched the search for the pharmacological effects of the chemical class of natural derivatives, phytocannabinoids. Now, over 500 compounds have been isolated from Cannabis sativa with approximately 105 being cannabinoids. ${ }^{5}$ Of those 105 compounds, 
THC has been determined to be the primary constituent; and it plays the central role in the discovery of a new signaling system in human body known as the endocannabinoid system. Two major cannabinoid receptors, $\mathrm{CB} 1$ (CB1R) and $\mathrm{CB} 2(\mathrm{CB} 2 \mathrm{R})$, were identified as mediators of the biological effects of THC. ${ }^{6}$ In addition, two lipid molecules, anandamide and 2-arachidonoyglycerol (2-AG), are recognized as being the endogenous ligands for these G-protein-coupled receptors (GPDRs). These, together with several metabolic enzymes and endogenous cannabinoids, constitute the endocannabinoid system. Numerous detailed research studies of the last three decades have confirmed the fundamental role of the endocannabinoid system in multiple physiological and pathophysiological processes. Most research has aimed to manage the activity of endocannabinoid pathways for medical therapeutic purposes.

CB1R has now been mapped in many species through autoradiographic methods, immuno histochemistry and in situ hybridization. CB1R is highly expressed in the brain, predominantly on neurons. The distribution of receptors is consistent between species and correlates well with the known behavioral effects of cannabinoids. Results show that cannabinoid receptor binding sites in the human brain are found mainly in the forebrain areas associated with higher cognitive functions; in the forebrain, midbrain and hindbrain areas associated with the control of movement; and in hindbrain areas associated with the control of motor and sensory functions of the autonomic nervous system. High levels of receptors are observed in the hippocampus, in basal ganglia output nuclei (globus pallidus and substantia nigra), the cerebellum, and cortical association regions, while moderate levels are observed in primary cortical regions, thalamic nuclei, and striatum, and low levels in brain stem and spinal cord regions. ${ }^{8}$ Retrograde activation of presynaptic CB1R on axon terminals is now considered the general signaling mode by which a postsynaptic neuron can temporarily or continuously limit the strength of its afferent inputs.

CB2Rs are located primarily on immune cells. Upon activation, they can modulate immune cell migration and cytokine release. ${ }^{8}$ Some neurons are expressing CB2Rs; however, the role of those neuron CB2Rs is not yet established.

More than three million Americans live with epilepsy. One third of these people have ongoing treatment resistant seizures. ${ }^{1}$ The anecdotal reports of the positive effects of the marijuana plant for some individuals with epilepsy are giving hope for all epilepsy patients and, especially, those with treatment-resistant epilepsy. Contemporary medical literature is filled with case reports on surveys of different cannabis products. Those reports have generally found that these products act as anticonvulsants in the majority of the cases. Proper randomized, double-blind, placebo-controlled trials are not permitted due to the fact that medical and recreational use of marijuana is still illegal in the many of the states in the U.S. and in most countries. Despite laws that permit use of medical marijuana in 40 states, cannabis is still illegal under federal law and is classified as a Schedule I drug. The federal government, therefore, views cannabis as highly addictive and having no medical value. ${ }^{10}$ Legal and ethical issues are making epidemiological studies on cannabis with high content of THC almost impossible. For now, researchers should consider present animal studies and case reports as valuable and useful sources of information regarding the potential benefits and limitations of cannabis treatment for seizures. These studies can indicate directions for cannabis research in the near future, especially when considering the strong association between high THC/low CBD-containing cannabis and the chances of first-episode psychosis. ${ }^{11}$
Another important consideration in this type of research is how long-term cannabis use for seizure control may affect the nervous system in epileptic patients upon cessation of marijuana smoking. A first, well-documented, case study described the reappearance of seizures in two patients whose focal epilepsy had previously been controlled by medical marijuana directly after stopping marijuana use. A dramatic increase in seizure frequency together with underlying abnormal electrical brain activity was documented with electroencephalogram video telemetry. ${ }^{12}$

Due to the high prevalence of childhood epilepsy, it is essential to research antiepileptic effects of cannabis. Russ et al..$^{13}$ performed cross-sectional analysis of data from the National Survey of Children's Health (2007) on 91,605 children ages birth to 17years, including 977 children reported by their parents to have been diagnosed with epilepsy/seizure disorder. ${ }^{13}$ Results showed that the estimated lifetime prevalence of epilepsy/seizure disorder was 10.2 per $1000(95 \%$ confidence interval) or 1\%; and current epilepsy/seizure disorder was 6.3 per 1000 (95\% confidence interval), or $0.6 \% .{ }^{13}$ Based on these findings, it is estimated that 466,000 patients in the U.S. and 765,000 childhood patients in Europe are currently diagnosed with epilepsy. Those numbers, together with the large numbers of refractory forms of epilepsy, make it imperative to find safe and efficient cannabinoidbased treatments. However, the concept of long-term administration of cannabis with higher concentration of THC is not favorable due to susceptibility in the developing prenatal, postnatal, and adolescent brain..$^{14}$ For instance, THC was reported to impair short-term memory and learning abilities, to cause neuronal cell death of the hippocampus after chronic exposure, and to increase the risk of developing psychotic disorders with maturity. ${ }^{15}$ Therefore, research efforts were focused on using cannabis enriched in CBD. This cannabis preparation showed anticonvulsant effects in multiple animal studies and in several preclinical and some clinical studies. ${ }^{16}$

The antiepileptic mechanisms of CBD are not known; it has little affinity for either CBR 1 or CBR2, ${ }^{17}$ but it has high antagonist effects at CBR1 and CBR2. ${ }^{18}$ However, this mechanism of CBD action cannot explain its antiepileptic properties because CBR1 activation is a primary protective measure against seizures. ${ }^{19}$ Furthermore, administration of low doses of THC or administration of synthetic cannabimimetics all anticonvulsant in vitro and in vivo; and these effects can be blocked by CBR1 antagonists. When applied alone, high doses of CBR1 antagonists have usually been shown to increase seizure duration and frequency. ${ }^{15}$ Mechanisms of action of CBD may include effects on the equilibrative nucleoside transporter; the orphan G-protein-coupled receptor GPR55; the transient receptor potential of melastatin type 8 channels; the 5-HT1A receptor; the $\alpha 3$ and $\alpha 1$ glycine receptors; and the transient receptor potential of ankyrin type 1 channel. CBD has neuroprotective and anti-inflammatory effects. ${ }^{20}$ It is not entirely clear how CBD works to control seizures; more research needed.

With the increasing legal availability of medical marijuana in some U.S. states, parents have started to use CBD cannabis preparations as an adjunctive therapy for their children who suffer from very serious and debilitating forms of childhood epilepsies. One of the State of Colorado's biggest cannabis success stories is the recent case report of Charlotte Figi, who suffers from Dravet syndrome (a severe form of epilepsy caused by a mutation in the SCN1A gene encoding a voltage-gated sodium channel subunit). Charlotte's seizures started at age 3 months as a prolonged status epilepticus. She had frequent febrile and afebrile status epilepticus as well as tonic, tonic-clonic, and myoclonic seizures. She began losing milestones, had significant 
cognitive and motor delays, required a feeding tube for nutrition and water, struggled to walk and talk, and required full assistance with her activities of daily living. At one point, she was experiencing up to 50 generalized tonic-clonic seizures per day. After unsuccessful treatment including all conventional anticonvulsant drugs (levetiracetam, oxcarbazepine, topiramate, zonisamide, valproate, clobazam, clonazepam, and valium) and the ketogenic diet, her parents tried CBD-enriched/low-THC content cannabis oil made from a specific cannabis strain, now named after her as Charlotte's Web (CW). This extract, slowly titrated over weeks and given sublingually in addition to her existing antiepileptic drug regimen, reduced Charlotte's seizure frequency to 2-3 nocturnal convulsions per month. This effect persisted for 20months, and Charlotte was successfully weaned from her other antiepileptic drugs. Then, her parents started to slowly decrease her $\mathrm{CW}$, and they reached $2 \mathrm{mg}$ of $\mathrm{CBD} / \mathrm{lb}$ per day (from her steady dose of $4 \mathrm{mg} \mathrm{CBD} / \mathrm{lb}$ per day). Charlotte's seizures started coming back. When she was completely off the $\mathrm{CW}$, her seizures returned to 5-10 seizures per day for 3days, at which time her parents restarted $\mathrm{CW}$. Now, Charlotte is feeding and drinking orally on her own, and her autistic behavior has lessened. She is finally able to walk and talk. Her seizure frequency is 2-3 nocturnal convulsions per month, a level consistently maintained over the last 3years. ${ }^{21}$

A survey organized by Stanford University, among parents who are using CBD cannabis preparations to treat their children with epilepsy, reported significant improvement in their children's condition. Nineteen children participated in this survey; they ranged in age from 2 to $16 y e a r s$. Thirteen children had Dravet syndrome, four children had Doose syndrome (epilepsy with myoclonic astatic seizures), and one each had Lennox-Gastaut syndrome (multiple different types of seizures, particularly tonic and atonic) and idiopathic early-onset epilepsy. In all cases, except one, the children experienced treatmentresistant epilepsy for more than 3years before trying CBD-enriched cannabis. The dosage of $\mathrm{CBD}$ that the parents reported providing ranged from less than $0.5 \mathrm{mg} / \mathrm{kg} /$ day to $28.6 \mathrm{mg} / \mathrm{kg} /$ day, whereas THC dosage was below $0.8 \mathrm{mg} / \mathrm{kg} /$ day in every case. The duration of CBDenriched cannabis administration ranged from two weeks to over one year. Sixteen $(84 \%)$ of the nineteen parents reported a reduction in their child's seizure frequency. Two parents reported that their child became seizure-free after more than 4months of CBD-enriched cannabis use. Three parents reported no change. Beneficial effects of CBD-enriched cannabis, other than reduced seizures, included better mood $(15 / 19$, $79 \%)$, increased alertness $(14 / 19,74 \%)$, better sleep $(13 / 19,68 \%)$ and decreased self-stimulation $(6 / 19,32 \%)$. Negative side effects included drowsiness $(7 / 19,37 \%)$ and fatigue $(3 / 19,16 \%){ }^{22}$

The new trend in medical cannabis use in children with epilepsy is raising significant safety issues. Cannabis extracts are often inaccurately labeled, and can contain highly variable levels of CBD and THC. Medical application of cannabis poses risks due to a lack of standardization and regulation, inexact dosing, possible adverse effects, and medication interactions. More valid data regarding the use of cannabis is coming from randomized clinical studies of CBDcontaining medications. A new drug Epidiolex (GW Pharmaceuticals) contains about $98 \%$ CBD with only trace amounts of other cannabinoids, and is approved as an investigational drug. In 2015, very promising results for Epidiolex were reported at the American Epilepsy Society Annual Meeting. ${ }^{23}$ In a prospective observational study, data was obtained from twenty five children with drug-resistant epilepsy. Patients were started on $5 \mathrm{mg} / \mathrm{kg} /$ day of CBD and increased weekly by $5 \mathrm{mg} / \mathrm{kg}$ increments until a maximum dose of $25 \mathrm{mg} / \mathrm{kg} /$ day was attained, in addition to antiepileptic drug therapy. The addition of $\mathrm{CBD}$ treatment was associated with more than a $50 \%$ seizure reduction in one third of the patients at 3months.

This improvement in seizure control was maintained in $40 \%$ of the patients for 12 months. The best results were obtained in Dravet syndrome patients. Twelve patients discontinued CBD due to lack of efficacy, and one patient discontinued due to a significant increase in seizures while on CBD treatment. ${ }^{23}$ More hope to patients with Lennox-Gastaut Syndrome is given by results of a phase 3 randomized, double-blind placebo-controlled trial on the same drug, Epidiolex; announced in June 2016. Data was obtained from 171 patients over a 14 week treatment period. Patients were age 2-55; the average age of trial participants was 15 years, and $34 \%$ were 18 years or older. Median reduction in monthly drop seizures of $44 \%$ was reported, compared with a reduction of $22 \%$ in patients given a placebo; the difference between treatments was statistically significant $(\mathrm{p}=0.0135) .{ }^{24}$ In addition, in March 2016, GW Pharmaceuticals announced positive results of the first pivotal phase 3 trial of Epidiolex in Dravet syndrome. ${ }^{24}$

\section{Conclusion}

Some evidence suggests that cannabis and cannabinoid-based medicines may be beneficial in some patient populations with epilepsy, but may not be effective in other populations; and aggravation of epileptic seizures can occur in some cases. Further research is needed. The American Epilepsy Society has stated: "At present, the epilepsy community does not know if marijuana is a safe and effective treatment. Nor do we know the long-term effects that marijuana will have on learning, memory, and behavior; especially in infants and young children." The American Epilepsy Society has also urged the U.S. Drug Enforcement Agency (DEA) to change marijuana from its current Schedule I status to allow researchers to conduct studies more efficiently. ${ }^{25}$

\section{Acknowledgements}

None.

\section{Conflict of interest statement}

The authors declare that the research was conducted in the absence of any commercial or financial relationships that could be construed as a potential conflict of interest.

\section{References}

1. American Epilepsy Society (2016).

2. http://www.nationalgeographic.com/magazine/2015/06/

3. Mechoulam R, Gaoni Y. The absolute conXiguration of $\delta 1$ tetrahydrocannabinol, the major active constituent of hashish. Tetrahedron Letters. 1967;8(12):1109-1111.

4. Mechoulam R, Shvo Y. Hashish-I:the structure of cannabidiol. Tetrahedron Letters. 1963;19(12):2073-2078.

5. Husni AS, McCurdy CR, Radwan MM, et al. Evaluation of phytocannabinoids from high potency using bioassays to determine structure-activity relationships for cannabinoid receptor 1 and cannabinoid receptor 2. Medicinal Chemistry Research. 2014;23(9):4295-4300.

6. LA Matsuda, Lolait SJ, Brownstein MJ, et al. Structure of a cannabinoid receptor and functional expression of the cloned cDNA. Nature. 1990;346(6284):561-564.

7. Blankman JL, Cravatt BF. Chemical probes of endocannabinoid metabolism. Pharmacol Rev. 2013;65(2):849-871. 
8. Glass M, Dragunow M, Faull M. Cannabinoid receptors in the human brain:A detailed anatomical and quantitative autoradiographic study in the fetal neonatal and adult human brain. Neuroscience. 1997;77(2):299 318 .

9. Castillo PE, Younts TJ, Cha'vez AE, et al. Endocannabinoid signaling and synaptic function. Neuron Review. 2012;76(1):70-81.

10. http://www.safeaccessnow.org/federal_marijuana_law

11. Di Forti M, Marconi A, Carra E, et al. Proportion of patients in south London with Xirst-episode psychosis attributable to use of high potency cannabis:A case-control study. Lancet Psychiatry. 2015;2(3):233-238.

12. Hegde M, Santos-Sanchez C, Hess CP, et al. Seizure exacerbation in two patients with focal epilepsy following marijuana cessation. Epilepsy Behav. 2012;25(4):563-566.

13. Russ SA, Larson K, Halfon N. A national proXile of childhood epilepsy and seizure disorder. Pediatrics. 2012;129(2):256-264.

14. Hadland SE, Knight JR, Harris SK. Medical marijuana:review of the science and implications for developmental-behavioral pediatric practice. J Dev Behav Pediatr. 2015;36(2):115-123.

15. Rudenko V, RaXiuddin A, Leheste JR, et al. Inverse relationship of cannabimimetic (Rp)WIN 55, 212 on behavior and seizure threshold during the juvenile period. Pharmacol Biochem Behav. 2012;100(3):474 484.

16. Jones NA, Glyn SE, Akiyama S, et al. Cannabidiol exerts anti-convulsant effects in animal models of temporal lobe and partial seizures. Seizure. 2012;21(5):344-352.
17. Bisogno T, Hanus L, De Petrocellis L, et al. Molecular targets for cannabidiol and its synthetic analogues: effect on vanilloid VR1 receptors and on the cellular uptake and enzymatic hydrolysis of anandamide. $\mathrm{Br} J$ Pharmacol. 2001;134(4):845-852.

18. Thomas A, Baillie GL, Phillips AM, et al. Cannabidiol displays unexpectedly high potency as an antagonist of $\mathrm{CB} 1$ and $\mathrm{CB} 2$ receptor agonists in vitro. Br J Pharmacol. 2007;150(5):613-623.

19. Citraro R, Russo E, Ngomba RT, et al. CB1 agonists, locally applied to the cortico-thalamic circuit of rats with genetic absence epilepsy, reduce epileptic manifestations. Epilepsy Res. 2013;106 (1-2):74-82.

20. Devinsky O, Cilio MR, Cross H, et al. Cannabidiol:Pharmacology and potential therapeutic role in epilepsy and other neuropsychiatric disorders. Epilepsia. 2014;55(6):791-802.

21. Maa E, Figi P. The case for medical marijuana in epilepsy. Epilepsia. 2014;55(6):783-786.

22. Porter BE, Jacobson C. Report of a parent survey of cannabidiolenriched cannabis use in pediatric treatment-resistant epilepsy. Epilepsy and Behavior. 2013;29(3):574-577.

23. Oldham M, Sullivan J, Singhal N, et al. Long term efXicacy and tolerability of add-on cannabidiol for drug resistant pediatric epilepsies. American Epilepsy Society. 2015.

24. GW Pharmaceuticals. GW Pharmaceuticals Announces Positive phase 3 Pivotal Trial Results for Epidiolex (cannabidiol) in the Treatment of Lennox-Gastaut Syndrome. UK: GW Pharmaceuticals; 2016.

25. American Epilepsy Society AES. AES Position on Medical Marijuana USA; 2016. 